\title{
Molecular line survey of NGC 7027
}

\author{
Yu-Chin Huang ${ }^{1,2}$, Dinh-V-Trung ${ }^{2}$ and Sun Kwok ${ }^{2,3}$ \\ ${ }^{1}$ Institute of Astrophysics, National Taiwan University, Taiwan \\ email: ychuang@asiaa.sinica.edu.tw \\ ${ }^{2}$ Institute of Astronomy and Astrophysics, Academia Sinica, Taiwan, \\ email:trung@asiaa.sinica.edu.tw \\ ${ }^{3}$ Department of Physics, University of Hong Kong, Hong Kong \\ email:sunkwok@hku.hk
}

\begin{abstract}
We report preliminary results of a spectral line survey of NGC 7027 in the $\lambda 3 \mathrm{~mm}$ and $1.3 \mathrm{~mm}$ bands using the Arizona Radio Observatory (ARO) 12-m and 10-m telescopes. To a sensitivity limit of $\sim 10-20 \mathrm{mK}$, we have detected 34 lines, including hydrogen recombination lines and rotational transitions of $\mathrm{CN}, \mathrm{CO}, \mathrm{CCH}, \mathrm{CO}^{+}, \mathrm{HCO}^{+}$, and their isotopes.
\end{abstract}

Keywords. planetary nebulae: invidual (NGC 7027), ISM: molecules

\section{Introduction}

Molecular envelopes of planetary nebulae (PNs) are an interesting laboratory for circumstellar chemistry as the processes of photodissociation, gas-phase and grain-phase chemical reactions can be observed over the short $\left(10^{3}\right)$ yr dynamical timescales of the envelope. The molecular envelope of NGC 7027 is particularly rich in molecular species. In addition to common molecular species such as $\mathrm{CO}$ and $\mathrm{HCN}$, various radicals (e.g., $\mathrm{C}_{2} \mathrm{H}$ ), rings (e.g., $\mathrm{C}_{3} \mathrm{H}_{2}$ ) and molecular ions (e.g., $\mathrm{HCO}^{+}$) have been observed (Deguchi et al. 1990, Jaminet et al. 1991, Hasegawa \& Kwok 2001). However, there has been no comprehensive spectroscopic survey of the molecular envelope of NGC 7027. Since April 2005, we have carried out a spectral line survey using the Arizona Radio Observatory 12-m and 10-m telescopes, with the aim to understand the chemical content of the circumstellar envelope around NGC 7027.

\section{Observations}

As of early 2006, we have covered part of the $\lambda 3 \mathrm{~mm}$ band (72-96 GHz) and most of the $\lambda 1 \mathrm{~mm}$ band (220-243 GHz and 246-266 GHz). The observations of the ARO 12-m were carried out using the filterbank and auto-correlator with bandwidths of $\sim 600 \mathrm{MHz}$. For the 10-m, the filterbank and acousto optical spectrometers were used as backends with bandwidths of $1 \mathrm{GHz}$. The spectral survey was carried out in tuning steps of 0.5 and $1 \mathrm{GHz}$ for the $12-\mathrm{m}$ and $10-\mathrm{m}$ observations, respectively. The system noise temperatures are typically $\sim 150-400 \mathrm{~K}$ at $\lambda 3 \mathrm{~mm}$ and $400-700 \mathrm{~K}$ at $\lambda 1 \mathrm{~mm}$. In order to achieve rms noise temperatures of $\sim 10 \mathrm{mK}$, integration times of $\sim 1-2$ hours are needed.

\section{Results}

A number of hydrogen recombination lines (H29-30 $\alpha, 41-44 \alpha)$ have been detected. An example of the 12-m spectrum showing the hydrogen recombination line $\mathrm{H} 44 \alpha$ at 74.64 $\mathrm{GHz}$ is shown in the top-left panel of Figure 1. On the top-right panel is a 10-m spectrum 

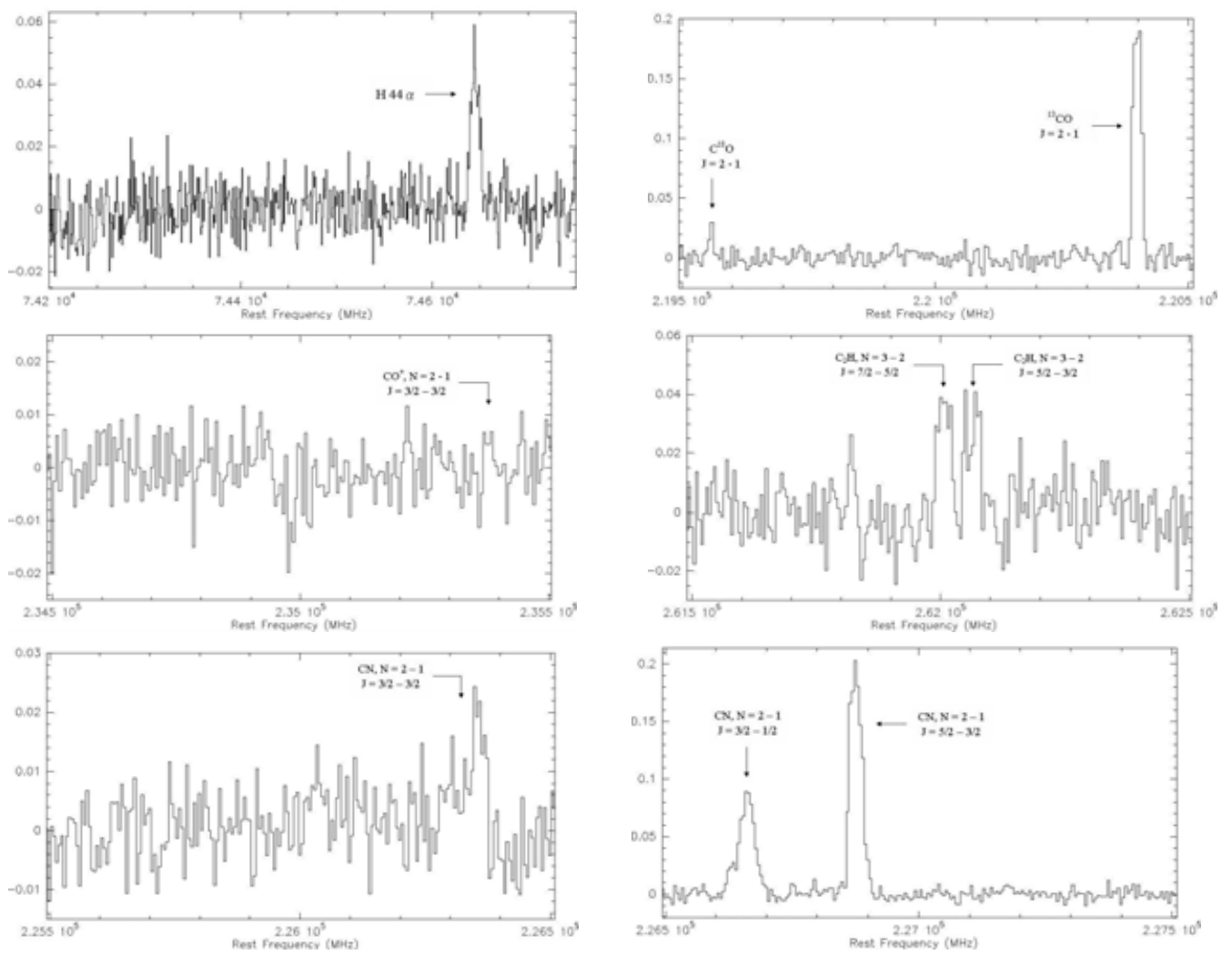

Figure 1. Examples of the survey spectra of NGC 7027 from the ARO 12-m and 10-m telescopes.

showing the ${ }^{13} \mathrm{CO} J=2-1$ line at $220.4 \mathrm{GHz}$ and $\mathrm{C}^{18} \mathrm{O} J=2-1$ line at $219.56 \mathrm{GHz}$. Other detected species in the survey include HCN $(J=1-0, F=1-1,2-1,0-1 ; J=$ $3-2), \mathrm{CO}(J=2-1), \mathrm{CO}^{+}(N=2-1), \mathrm{CCH}(N=3-2)$, (Fig. 1, middle row), and different hyperfine transitions of $\mathrm{CN}(N=2-1$, Fig. 1, bottom row). A total of 34 lines have been detected, including several unidentified lines (e.g., at $221.462 \mathrm{GHz}$ ). Since the line width of NGC 7027 is about $30-40 \mathrm{~km} \mathrm{~s}^{-1}$ due to the expansion of the envelope, molecular transitions from different species are often blended together.

\section{Conclusions}

A spectral survey of this kind will allow us to determine molecular abundances in a uniform manner. While the recombination lines probe the ionized region, the molecular ions and neutral molecules will probe the photodissociation region and the molecular regions respectively. The kinetic temperature can be determined from ratios of the strengths of different rotational transitions. These results will be compared to our other ongoing spectral surveys of AGB stars (IRC+10216) and proto-planetary nebulae (AFGL 2688).

\section{References}

Deguchi, S., Izumiura, H., Kaifu, N., Mao, X., Nguyen-Q-Rieu, \& Ukita, N. 1990, ApJ 351, 522 Hasegawa, T. \& Kwok, S. 2001, ApJ 562, 824

Jaminet, P. A., Danchi, W. C., \& Sutton, E. C. 1991, ApJ 380, 461 